\title{
Integrated Nutrient Management for Yield and Economics of Maize (Zea mays L.) In-Rice-Gingelly-Maize Cropping System through Integrated Farming System
}

\author{
K. Maruthupandi* and C. Jayanthi \\ Department of Agronomy, Tamil Nadu Agricultural University, \\ Coimbatore-641003, Tamil Nadu, India \\ *Corresponding author
}

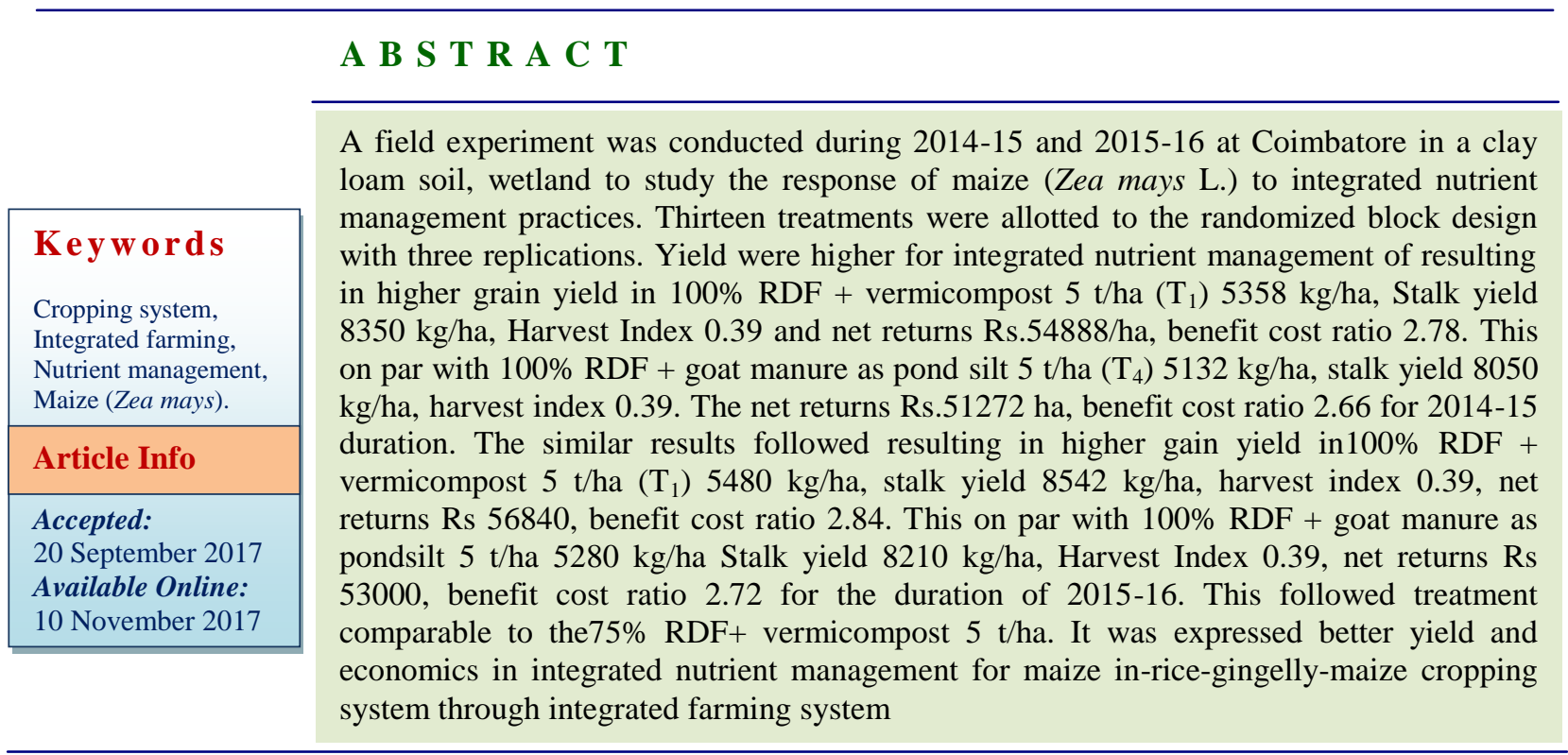

\section{Introduction}

Maize (Zea mays L.) is an important one in rice-gingelly-maize cropping system of Southern India. The productivity of this system, as the maize crops are high nutrientrequiring ones and respond well to higher levels of chemical fertilizers. But deterioration in soil health associated with global crises of energy, escalation in the prices of chemical fertilizers and environmental hazards due to excessive use of fertilizers, lead to emphasize on supplementation or substitution of chemical fertilizers with low priced nutrient sources such as organic and biosources. Application of these nutrient sources alone or in combination with inorganic, organic sources had been found beneficial not only in enhancing the productivity of maize in the cropping system (Jamwal, 2005), but also had the beneficial impact on soil properties (Pathak et al., 2005). The beneficial effect of organic sources applied in preceding crops was recorded in succeeding maize crop 
(Yadav et al., 2005, Yadav et al., 2008). The carry over effect of fertilizers and manures applied to rice-gigelly had also been reported in maize (Jamwal, 2005, Kumar and Ahlawat, 2004, Tiwari et al., 2004). However, on the fertility after rice-gingelly, the full yield potential of improved because of their integrated nutrient management. Therefore, an attempt was made to study the effects of inorganic and organic fertilizers on the productivity and soil properties of maize by rice-gingelly-maize cropping system through integrated farming system.

\section{Materials and Methods}

The experiment was conducted at the wetland farm, Tamil Nadu Agricultural university, Coimbatore, Tamil Nadu situated at 11' $\mathrm{N}$ latitude, $77^{\prime} \mathrm{E}$ longitude and 426.7 meters above mean sea level during the rabi seasons of 2014-2015 and 2015-2016. The soil of the experimental site was clay loam in texture with $\mathrm{pH} 8.42$ organic carbon $5.4 \mathrm{~g} / \mathrm{kg}$ and available N, P and K, 310, 12.9 and 712 $\mathrm{kg} / \mathrm{ha}$. The total amounts of rainfall received during the cropping seasons were 198.4 and $163.7 \mathrm{~mm}$, respectively. The mean maximum and minimum temperatures of both the seasons were within the optimal range of 27.6, 36.2 $0 \mathrm{C}$ and 18.1, 25.5 0C, 27.3, 37.9 and 16.7, 26.0 $0 \mathrm{C}$ in 2014-15 and 2015-16, respectively. The experiment was laid out in a randomized block design with three replications and thirteen treatments. The physic chemical characteristics according to the methods as described measurements are presented in Table 1. Vermicompost, FYM, Turkey manure as pond silt, Quail manure as pond silt and Goat manure as pond silt used as organic manure for field application and inorganic fertilizer. Organic manure was applied before transplanting of rice only. Treatment details wise applied (Table 2). As regards to fertilizers, was applied basal. Maize (COH6) @15 kg seed/ha was sown at
$60 \times 25 \mathrm{~cm}$ spacing. Observations on crop yield were recorded yield Parameters. The economics was calculated as per the official market price for different commodities.

\section{Results and Discussion}

\section{Grain yield, stalk yield, harvest index}

Significant variation in grain and stover yields and harvest index of maize was recorded due to nutrient management practices in both the years (Table 3). Higher grain yield in $100 \%$ RDF + vermicompost 5 t/ha (T1) $5358 \mathrm{~kg} / \mathrm{ha}$, Stalk yield $8350 \mathrm{~kg} / \mathrm{ha}$, Harvest Index 0.39 was recorded as compared to other treatments. However, different integrated nutrient management treatments did not differ with each other during the first year. During second year application higher gain yield in $100 \% \mathrm{RDF}+$ vermicompost $5 \mathrm{t} / \mathrm{ha}$ (T1) $5480 \mathrm{~kg} / \mathrm{ha}$, Stalk yield $8542 \mathrm{~kg} / \mathrm{ha}$, Harvest Index 0.39 was at par with recommended dose of the $75 \% \mathrm{RDF}+$ vermicompost $5 \mathrm{t} / \mathrm{ha}$. This was attributed to the better growth to yield parameters on the same nutrient management treatments. (Table 3) Mazzonccini et al., (2008) also reported the similar results the grain and straw yield of maize recorded the highest value in both the years. In general, grain and straw yields were higher during second year in comparison to first year and the increase in yields was higher in the treatments of combined use of different nutrients sources. The results are in agreement with that of Kumar and Ahlawat (2004) and Kumar (2008).

\section{Economics}

The cost of cultivation/ha of maize varied from Rs 27710 to Rs 30840 for both the years (Table 4). The treatments comprising organic sources of nutrients had higher cost than the fertilizer based treatment 100\% RDF + vermicompost 5 t/ha (T1) due to higher cost 
of vermicompost, manure as pond silt, farmyard manure than inorganic fertilizer. However, net returns (Rs 54888 and Rs 56840/ha) and net returns/rupee (Rs 2.78 and Rs 2.84) were highest with the application of $100 \% \mathrm{RDF}+$ vermicompost $5 \mathrm{t} / \mathrm{ha}$, while the treatment $50 \% \mathrm{RDF}+$ quail manure as pond silt $5 \mathrm{t} / \mathrm{ha} \mathrm{t}$ recorded lowest values of net returns and net returns/rupee invested. Similar findings were agreement with that of Bellakki (1998) and Yadav (2008).

\begin{tabular}{|l|c|c|}
\hline \multicolumn{3}{|c|}{ Table.1 Physico chemical characteristics of the experimental field } \\
\hline \multicolumn{1}{|c|}{ 1.Physical properties } & Units & Quantity \\
\hline a. Mechanical analysis & $(\%)$ & 36.40 \\
\hline 1. Sand & $(\%)$ & 19.20 \\
\hline 2. Silt & $(\%)$ & 44.10 \\
\hline 3. Clay \\
\hline 2. Chemical properties \\
\hline a. $\mathrm{pH}(1: 2.5$ soil/water $)$ & - & 8.42 \\
\hline b. Electric conductivity & $(\mathrm{ds} / \mathrm{m})$ & 0.39 \\
\hline c. Organic carbon & $(\mathrm{Kg} / \mathrm{ha})$ & 0.54 \\
\hline d. Available Nitrogen & $(\mathrm{Kg} / \mathrm{ha})$ & 30 \\
\hline e. Bray-P(Kg ha $\left.{ }^{-1}\right)$ & $(\mathrm{Kg} / \mathrm{ha})$ & 12.90 \\
\hline f. $\mathrm{NH}_{4} \mathrm{OAC}-\mathrm{K}$ & $(\mathrm{Kg} / \mathrm{ha})$ & 712 \\
\hline
\end{tabular}

\begin{tabular}{|l|l|}
\hline \multicolumn{2}{|c|}{ Table.2 Treatments details } \\
\hline $\mathbf{T}_{\mathbf{1}}$ & 100\% RDF + vermicompost $5 \mathrm{t} / \mathrm{ha}$ \\
\hline $\mathbf{T}_{\mathbf{2}}$ & $100 \% \mathrm{RDF}+$ turkey manure as pondsilt $5 \mathrm{t} / \mathrm{ha}$ \\
\hline $\mathbf{T}_{\mathbf{3}}$ & $100 \% \mathrm{RDF}+$ quail manure as pondsilt $5 \mathrm{t} / \mathrm{ha}$ \\
\hline $\mathbf{T}_{\mathbf{4}}$ & $100 \% \mathrm{RDF}+$ goat manure as pondsilt $5 \mathrm{t} / \mathrm{ha}$ \\
\hline $\mathbf{T}_{\mathbf{5}}$ & $75 \% \mathrm{RDF}+$ vermicompost $5 \mathrm{t} / \mathrm{ha}$ \\
\hline $\mathbf{T}_{\mathbf{6}}$ & $75 \% \mathrm{RDF}+$ turkey manure as pondsilt $5 \mathrm{t} / \mathrm{ha}$ \\
\hline $\mathbf{T}_{\mathbf{7}}$ & $75 \% \mathrm{RDF}+$ quail manure as pondsilt $5 \mathrm{t} / \mathrm{ha}$ \\
\hline $\mathbf{T}_{\mathbf{8}}$ & $75 \% \mathrm{RDF}+$ goat manure as pondsilt $5 \mathrm{t} / \mathrm{ha}$ \\
\hline $\mathbf{T}_{\mathbf{9}}$ & $50 \% \mathrm{RDF}+$ vermicompost $5 \mathrm{t} / \mathrm{ha}$ \\
\hline $\mathbf{T}_{\mathbf{1 0}}$ & $50 \% \mathrm{RDF}+$ turkey manure as pondsilt $5 \mathrm{t} / \mathrm{ha}$ \\
\hline $\mathbf{T}_{\mathbf{1 1}}$ & $50 \% \mathrm{RDF}+$ quail manure as pondsilt $5 \mathrm{t} / \mathrm{ha}$ \\
\hline $\mathbf{T}_{\mathbf{1 2}}$ & $50 \% \mathrm{RDF}+$ goat manure as pondsilt $5 \mathrm{t} / \mathrm{ha}$ \\
\hline $\mathbf{T}_{\mathbf{1 3}}$ & $\mathrm{RDF}+\mathrm{FYM} 12.5 \mathrm{t} / \mathrm{ha}$ \\
\hline & $\mathrm{RDF}:$ As per the recommendation to individual crop \\
\hline & OM $:$ First crop in the system \\
\hline
\end{tabular}


Table.3 Effect of integrated nutrient management on grain yield $(\mathrm{kg} / \mathrm{ha})$ and stover yield $(\mathrm{kg} / \mathrm{ha})$ and harvest index of maize in ricegingelly-maize cropping system

\begin{tabular}{|c|c|c|c|c|c|c|}
\hline \multirow[b]{2}{*}{ Treatment } & \multicolumn{3}{|c|}{ 2014-2015 } & \multicolumn{3}{|c|}{ 2015-2016 } \\
\hline & $\begin{array}{c}\text { Grain } \\
\text { yield } \\
\text { (kg/ha) }\end{array}$ & $\begin{array}{c}\text { Stover } \\
\text { yield } \\
\text { (kg/ha) }\end{array}$ & $\begin{array}{c}\text { Harvest } \\
\text { Index }\end{array}$ & $\begin{array}{c}\text { Grain } \\
\text { yield } \\
\text { (kg/ha) }\end{array}$ & $\begin{array}{c}\text { Stover } \\
\text { yield } \\
\text { (kg/ha) }\end{array}$ & $\begin{array}{c}\text { Harvest } \\
\text { Index }\end{array}$ \\
\hline $\mathrm{T}_{1}-100 \% \mathrm{RDF}+$ vermicompost & 5358 & 8350 & 0.39 & 5480 & 8542 & 0.39 \\
\hline$T_{2}-100 \%$ RDF + turkey manure as pondsilt & 4798 & 7956 & 0.38 & 5120 & 8106 & 0.39 \\
\hline$T_{3}-100 \%$ RDF + quail manure as pondsilt & 4614 & 7865 & 0.37 & 5036 & 7924 & 0.39 \\
\hline $\mathrm{T}_{4}-100 \% \mathrm{RDF}+$ goat manure as pondsilt & 5132 & 8050 & 0.39 & 5240 & 8210 & 0.39 \\
\hline $\mathrm{T}_{5}-75 \% \mathrm{RDF}+$ vermicompost & 5004 & 8150 & 0.38 & 5180 & 8360 & 0.38 \\
\hline$T_{6}-75 \%$ RDF + turkey manure as pondsilt & 4458 & 7685 & 0.37 & 4680 & 7895 & 0.37 \\
\hline $\mathrm{T}_{7}-75 \% \mathrm{RDF}+$ quail manure as pondsilt & 4174 & 7458 & 0.36 & 4356 & 7668 & 0.36 \\
\hline $\mathrm{T}_{8}-75 \% \mathrm{RDF}+$ goat manure as pondsilt & 4688 & 7892 & 0.37 & 4822 & 8102 & 0.37 \\
\hline $\mathrm{T}_{9}-50 \% \mathrm{RDF}+$ vermicompost & 4388 & 7545 & 0.37 & 4660 & 7755 & 0.38 \\
\hline $\mathrm{T}_{10}-\mathbf{5 0 \%} \mathrm{RDF}+$ turkey manure as pondsilt & 3878 & 7175 & 0.35 & 3930 & 7385 & 0.35 \\
\hline $\mathrm{T}_{11}-50 \% \mathrm{RDF}+$ quail manure as pondsilt & 3588 & 6817 & 0.34 & 3710 & 7027 & 0.35 \\
\hline$T_{12}-50 \%$ RDF + goat manure as pondsilt & 4218 & 7450 & 0.36 & 4340 & 7560 & 0.36 \\
\hline $\mathrm{T}_{13}-100 \% \mathrm{RDF}+\mathrm{FYM}$ at $12.5 \mathrm{t} / \mathrm{ha}$ & 4838 & 7880 & 0.38 & 4932 & 8090 & 0.38 \\
\hline SEd & 251 & 407 & 0.20 & 264 & 425 & 0.20 \\
\hline CD $(P=0.05)$ & 518 & 820 & 0.041 & 532 & 856 & 0.042 \\
\hline
\end{tabular}

$\mathrm{T}_{1}$ to $\mathrm{T}_{12}$ - Organic manure at $5 \mathrm{t} / \mathrm{ha}$; DAS - Days after sowing 
Table.4 Integrated nutrient management on economics of maize in rice-gingelly-maize cropping system

\begin{tabular}{|c|c|c|c|c|c|c|c|c|}
\hline \multirow[b]{2}{*}{ Treatment } & \multicolumn{4}{|c|}{ 2014-2015 } & \multicolumn{4}{|c|}{ 2015-2016 } \\
\hline & $\begin{array}{c}\text { Cost } \\
\text { of } \\
\text { Cultivation } \\
\end{array}$ & $\begin{array}{c}\text { Gross } \\
\text { return } \\
\text { (Rs/ha) }\end{array}$ & $\begin{array}{c}\text { Net } \\
\text { return } \\
(\mathrm{Rs} / \mathrm{ha})\end{array}$ & $\begin{array}{c}\text { B:C } \\
\text { ratio } \\
\text { (Rs/ha) }\end{array}$ & $\begin{array}{c}\text { Cost } \\
\text { of } \\
\text { Cultivation } \\
\end{array}$ & $\begin{array}{c}\text { Gross } \\
\text { return } \\
\text { (Rs/ha) }\end{array}$ & $\begin{array}{c}\text { Net } \\
\text { return } \\
(\mathrm{Rs} / \mathrm{ha})\end{array}$ & $\begin{array}{c}\text { B:C } \\
\text { ratio } \\
(\mathbf{R s} / \mathbf{h a})\end{array}$ \\
\hline$T_{1}-100 \% R D F+$ vermicompost & 30840 & 85728 & 54888 & 2.78 & 30840 & 87680 & 56840 & 2.84 \\
\hline$T_{2-100 \%}$ RDF + turkey manure as pondsilt & 30840 & 76768 & 45928 & 2.49 & 30840 & 81920 & 51080 & 2.66 \\
\hline$T_{3}-100 \%$ RDF + quail manure as pondsilt & 30840 & 73824 & 42984 & 2.39 & 30840 & 80576 & 49736 & 2.61 \\
\hline $\mathrm{T}_{4}-100 \% \mathrm{RDF}+$ goat manure as pondsilt & 30840 & 82112 & 51272 & 2.66 & 30840 & 83840 & 53000 & 2.72 \\
\hline $\mathrm{T}_{5}-75 \% \mathrm{RDF}+$ vermicompost & 29790 & 80064 & 50274 & 2.69 & 29790 & 82880 & 53090 & 2.78 \\
\hline$T_{6}-75 \%$ RDF + turkey manure as pondsilt & 29790 & 71328 & 41538 & 2.39 & 29790 & 74880 & 45090 & 2.51 \\
\hline $\mathrm{T}_{7}-75 \% \mathrm{RDF}+$ quail manure as pondsilt & 29790 & 66784 & 36994 & 2.24 & 29790 & 69696 & 39906 & 2.34 \\
\hline $\mathrm{T}_{8}-75 \% \mathrm{RDF}+$ goat manure as pondsilt & 29790 & 75008 & 45218 & 2.52 & 29790 & 77152 & 47362 & 2.59 \\
\hline $\mathrm{T}_{9}-50 \% \mathrm{RDF}+$ vermicompost & 27710 & 70208 & 42498 & 2.53 & 27710 & 74560 & 46850 & 2.69 \\
\hline $\mathrm{T}_{10^{-}} \mathbf{5 0 \%} \mathrm{RDF}+$ turkey manure as pondsilt & 27710 & 62048 & 34338 & 2.24 & 27710 & 62880 & 35170 & 2.27 \\
\hline$T_{11}-50 \%$ RDF + quail manure as pondsilt & 27710 & 57408 & 29698 & 2.07 & 27710 & 59360 & 31650 & 2.14 \\
\hline$T_{12}-50 \%$ RDF + goat manure as pondsilt & 27710 & 67488 & 39778 & 2.44 & 27710 & 69440 & 41730 & 2.51 \\
\hline$T_{13}-100 \%$ RDF + FYM at 12.5 t/ha & 30840 & 77408 & 46568 & 2.51 & 30840 & 78912 & 48072 & 2.56 \\
\hline
\end{tabular}

$\mathrm{T}_{1}$ to $\mathrm{T}_{12}$ - Organic manure at $5 \mathrm{t} / \mathrm{ha}$; Data not analysed 
For getting the better productivity and returns from maize in cropping system, $100 \% \mathrm{RDF}+$ vermicompost $5 \mathrm{t} / \mathrm{ha}$ (T1), followed by application of $100 \% \mathrm{RDF}+$ goat manure as pond silt $5 \mathrm{t} / \mathrm{ha}$ (T4), which resulted in $25 \%$ NPK saving in further cropping sequence period and the rice-gingelly-maize cropping system to reduce fertilizer usage and optimization followed in fertilizer utility to can derive sustainability.

\section{References}

Bellakki M.A, V.P. Bahadur and Setty, R.A. 1998. Effect of long term integrated nutrient management on some important properties of a vertisol. Journal of Indian Society of Soil Sciences 46: 176180.

Jamwal J.S. 2005. Productivity and economics of maize (Zea mays) - wheat (Triticum aestivum) cropping system under integrated nutrient supply system in rainfed areas of Jammu. Indian Journal of Agronomy 50 (2): 110-12.

Kumar Ashok. 2008. Direct and residual effect of nutrient management in maize (Zea mays L.) - wheat (Triticum aestivum L.) cropping system. Indian Journal of Agronomy 53 (1): 3 - 41.

Kumar, V. and Ahlawat, I.P.S. 2004. Carry over effect of biofertilizers and nitrogen applied to wheat (Triticum aestivum L.) and direct applied $\mathrm{N}$ in maize (Zea mays L.) in wheat - maize cropping system. Indian Journal of Agronomy 49 (4): 233-6.
Mazzoncini, M., P. Migliorini, D. Antichi and Vazzana, C. 2008. Effects of green manure and organic fertilizers on organic maize (Zea mays L.) in south Tuscany. Poster presented at cultivating the Future based on science: 2nd Conference of the International Society of Organic Agriculture Research ISOFAR, Modena held at Italy during 18-20 June, 2008.

Pathak, S.K., S.B. Singh, R.N. Jha and Sharma, R.P. 2005. Effect of nutrient management on nutrient uptake and changes in soil fertility in maize (Zea mays) - wheat (Triticum aestivum) cropping system. Indian Journal of Agronomy 50(4): 269 - 73.

Tiwari, R.C., P.K. Sharma and Khandelwal, S.K. 2004. Effect of green manuring through Sesbania cannabina and Sesbania rostrata and nitrogen application through urea to maize (Zea mays) - wheat (Triticum aestivum) cropping system. Indian Journal of Agronomy 49(1): 15-21.

Yadav, M.P., M. Aslam and Kushwaha, S.P. 2005. Effect of integrated nutrient management on rice (Oryza sativa)wheat (Triticum aestivum) cropping system in Central Plains Zone of Uttar Pradesh. Indian Journal of Agronomy 50(2): 89-93.

Yadav, R.L, D.V. Yadav and Duttamajumdar, S.K. 2008. Rhizospheric environment and crop productivity: A review. Indian Journal of Agronomy 53(1): 1-17. 23

\section{How to cite this article:}

Maruthupandi, K. and Jayanthi, C. 2017. Integrated Nutrient Management for Yield and Economics of Maize (Zea mays L.) In-Rice-Gingelly-Maize Cropping System through Integrated Farming System. Int.J.Curr.Microbiol.App.Sci. 6(11): 2745-2750. doi: https://doi.org/10.20546/ijcmas.2017.611.324 